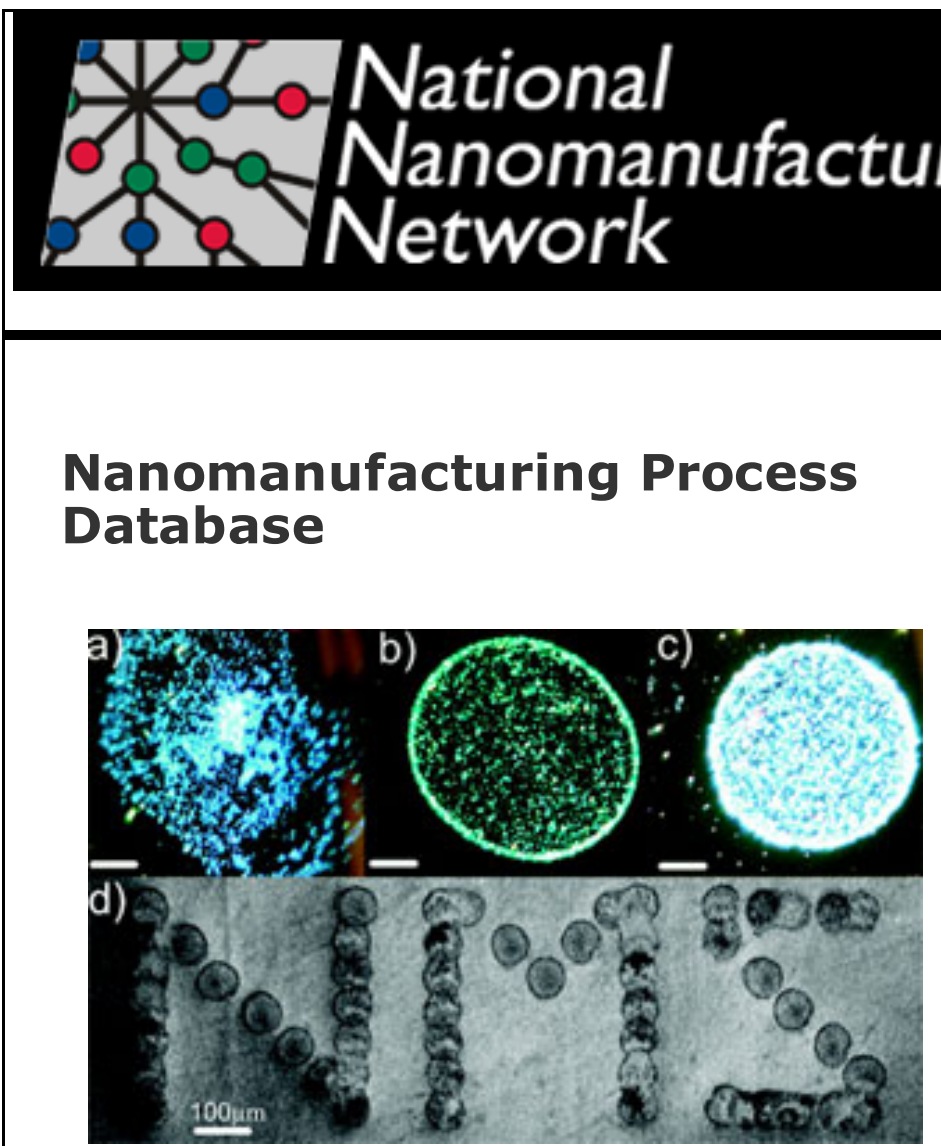

Nanomanufacturing processes are typically unique methods employed to control the shape, features, size, and morphology of materials in order to achieve some value-added functionality. Depending on the targeted objectives, numerous approaches and materials could be explored to reach the final goals. While such process research and development may be exciting and educational to researchers, often times this results in extensive cost and time delays in reaching a scalable nanomanufacturing path that could lead to commercialization of nanotechnology-enabled products. This is of particular importance to industry where it is critical to identify and optimize the nanomanufacturing process development cycle, enabling a more competitive innovation ecosystem. As an added resource in nanomanufacturing process $R \& D$, InterNano hosts a small but growing Process Database, or a knowledge base of techniques for processing nanoscale materials, devices, and structures. The entries include stepby-step descriptions, images, notes on methodology and environmental variables, and associated references and patent information. The purpose of the Process Database is to enable sharing of appropriate process knowledge across laboratories. The National Nanomanufacturing Network (NNN) is working to facilitate the access to
Volume 5 Issue 9 - September 2012

\section{Job Opportunities}

Assistant Professor in Mechanical Engineering Missouri University of Science and Technology

Corporate \& Outreach Manager Northeastern University

Physical Scientist NIST

\section{Upcoming Events}

October 4-7, 2012

2012 IEEE Workshop on

Nanoinformatics for Biomedicine

October 9, 2012

Demystifying Nano

October 25-26, 2012

EU-US Bridging NanoEHS

Research Efforts - A Joint

Workshop 2012

View Full Calendar

\section{Upcoming Calls}


and documentation of a rich and representative collection of nanomanufacturing processes, and encourages the nanomanufacturing community to contribute to this collection. More....

Regards,

Jeff Morse, Managing Director,

National Nanomanufacturing Network

\section{Learn More about the Nanomanufacturing}

\section{Nanotech Security Achieves High Speed Production Run of Metallized Optical Images}

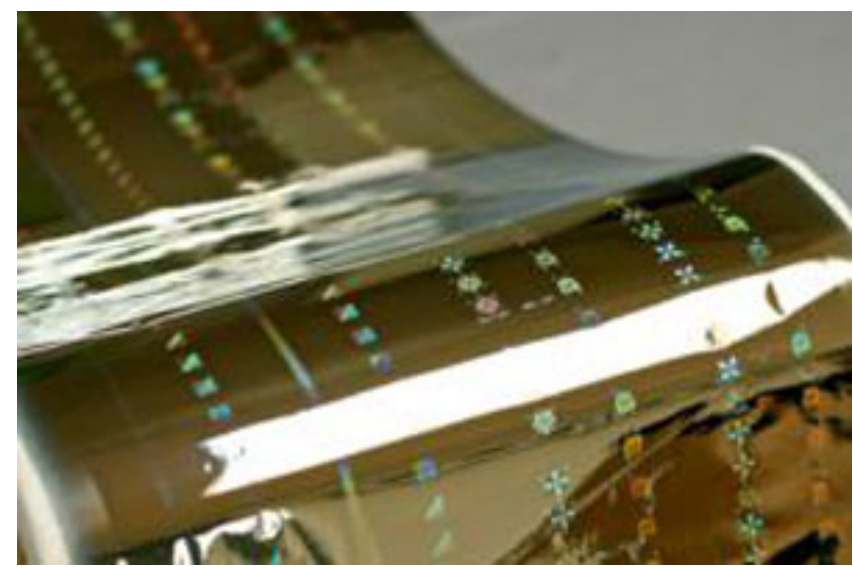

Nanotech Security Corp., developer of nextgeneration security and authentication features using nanotechnology based optics, announced that it has achieved a technological breakthrough with the successful metallization of images in a commercial-scale run. The 1500 foot length of optical images embossed on a 10 inch metallized roll was produced by an independent third party as part of its feasibility due diligence review of the technology. This milestone shows that Nanotech's advanced optical technology can be seamlessly incorporated into standard security industry manufacturing processes.

The third party which conducted the test-run is the same billion dollar company which conducted the first commercial scale run several months ago using clear polymer. The optical roll was produced using conventional roll-to-roll printing technology while being metallized using rapid evaporation of aluminum to produce a highly reflective thin film surface. Metallization of the roll creates a vibrant, crisp, ultra high definition image and security feature. Nanotech designed and manufactured the original set of master shims provided to the third party for the commercial scale trial. NOtES $\otimes$ is 12th Annual Flexible Electronics

\& Displays Conference \&

Exhibition

Submissions accepted until:

October 5, 2012

Call for User Proposals: HighImpact Nanoscience Research Center for Nanophase

Materials Sciences Oak Ridge National Laboratory

Submissions accepted until:

October 17, 2012

International Conference on Material Science (ICMS2013) Submissions accepted until: October 31, 2012

View All Calls

Advertisements

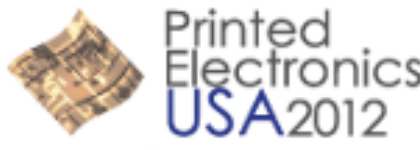

December 5-6, 2012 Santa Clara, CA, USA

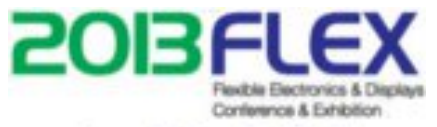

Jan 29 - Feb 1

Phoenix, Arizona Flextechisuner

\section{Recently Published}

From Our Affiliates

Lack of cytochrome involvement in long-range electron transport through conductive biofilms and nanowires of Geobacter sulfurreducens Energy \& Environmental Science 5(9): 8651-8659

Silicon nanomembranes for fingertip electronics Nanotechnology 23(34)

Analyzing High Dimensional Toxicogenomic Data Using Consensus Clustering 
Nanotech's sophisticated authentication feature which replicates nanoscale structures similar to those found on the wings of the iridescent blue Morpho Butterfly.

More...

\section{Nanotechnology-enabled Smart Packaging for Longer Produce Shelf-life}

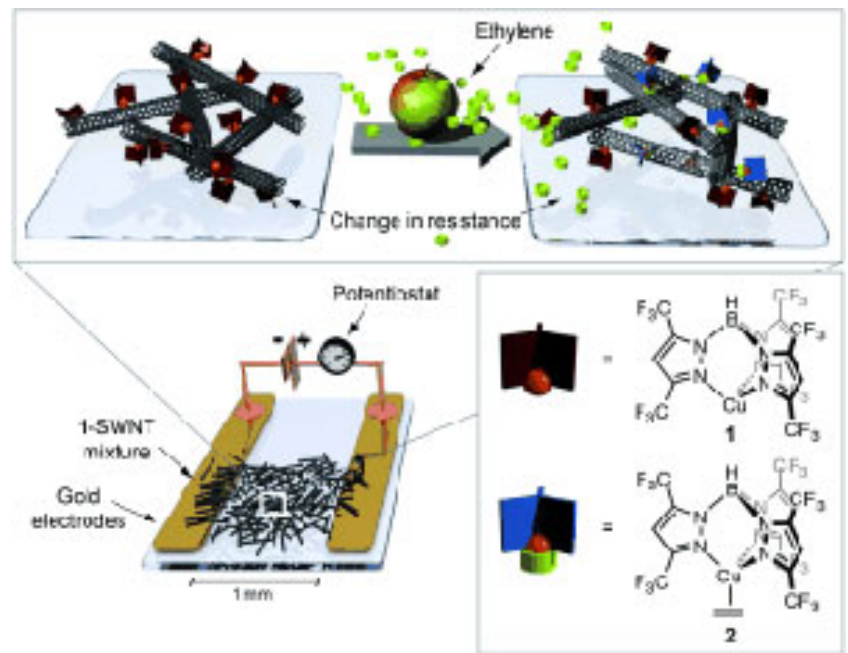

Horticultural industries face significant challenges in determining the optimal time for harvesting, storage, and transport of produce in order to avoid substantial degradation and waste due to overripening. The culprit for this is ethylene, the smallest of plant hormones, which plays a key role in many plant developmental processes, including the ripening of fruits and vegetables. The ripening process is the result of ethylene binding to the receptor ETR1, leading to the translation of ripening genes, and subsequently the production of enzymes that induce the visible signs of ripening. Measures of the internal ethylene concentration in fruit, for example, can provide an indicator to determine the time to harvest, and monitoring of ethylene levels during storage and transportation can avoid overripening of the fruit. Additionally, methods to scavenge ethylene could extend the shelf-life of produce.

More....

\section{Researchers Report Novel Approach for Single Molecule Electronic DNA Sequencing}

Environmental Science \&

Technology 46(15): 8413-8421

Electrical Conductivity in a

Mixed-Species Biofilm

Applied and Environmental

Microbiology 78(16): 59675971

Real-time optimal commutation for minimizing thermally induced inaccuracy in multimotor driven stages Automatica 48(8)

\section{Affiliated Centers}
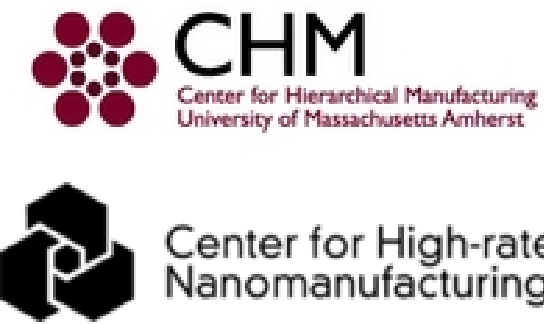

Center for High-rate Nanomanufacturing

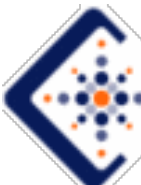

Center for Nanoscale Chemical-Electrical-Mechanical Manufacturing Systems
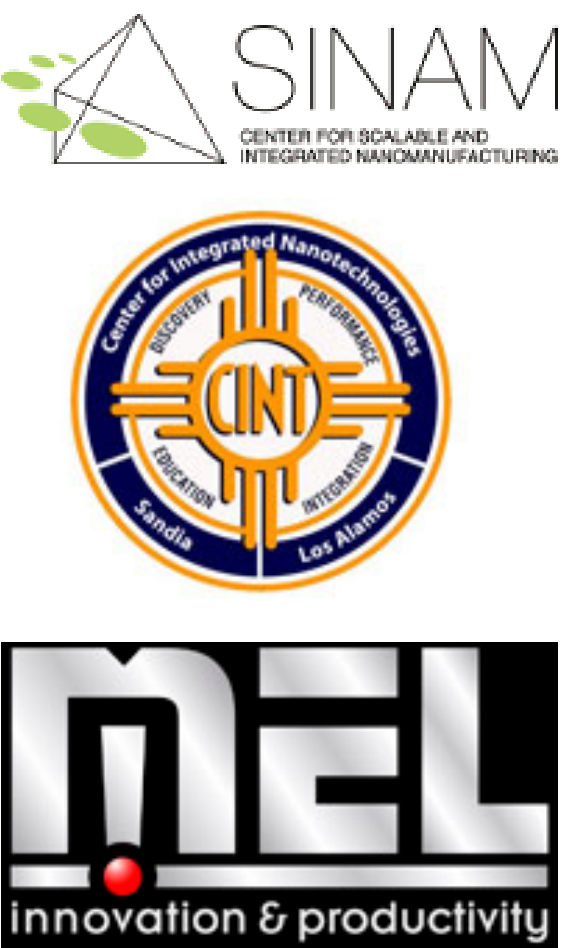


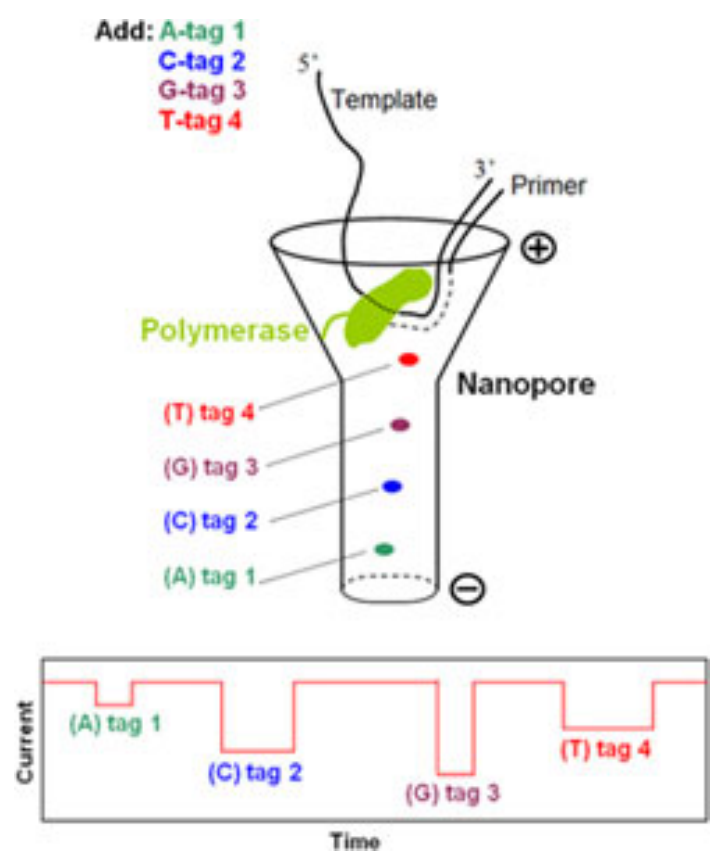

DNA sequencing is the driving force behind key discoveries in medicine and biology. For instance, the complete sequence of an individual's genome provides important markers and guidelines for medical diagnostics and healthcare. Up to now, the major roadblock has been the cost and speed of obtaining highly accurate DNA sequences. While numerous advances have been made in the last 10 years, most current high-throughput sequencing instruments depend on optical techniques for the detection of the four building blocks of DNA: A, C, G and $T$. To further advance the measurement capability, electronic DNA sequencing of an ensemble of DNA templates has also been developed. Recently, it has been shown that DNA can be threaded through protein nanoscale pores under an applied electric current to produce electronic signals at single molecule level. However, because the four nucleotides are very similar in their chemical structures, they cannot easily be distinguished using this technique. Thus, the research and development of a single-molecule electronic DNA sequencing platform is the most active area of investigation and has the potential to produce a hand-held DNA sequencer capable of deciphering the genome for personalized medicine and basic biomedical research.

More....

\section{Read more on InterNano}


Subscribe / Unsubscribe from this list.

Our mailing address is:

The National Nanomanufacturing Network

374 Lederle Graduate Research Center

710 N. Pleasant Street

University of Massachusetts

Amherst, MA 01003

Our email address is:

nnn@nanomanufacturing.org

Our phone number is:

(413) 577-0570

Copyright (C) 2012 The National Nanomanufacturing Network All rights reserved.

Supported by the National Science Foundation under Grant No. CMMI-1025020. 